\title{
Taste preferences as a function of food deprivation during original taste exposure
}

\author{
ELIZABETH D. CAPALDI and DAVID E. MYERS \\ Purdue University, West Lafayette, Indiana 47907
}

\begin{abstract}
It has been reported previously that rats prefer a flavor they consumed under high deprivation to a flavor they consumed under low deprivation (Revusky, 1967). Here it was found that this preference occurs only if nutritive solutions are used and the flavors are given preceding and following eating. If flavors are given separately from the daily feeding, rats prefer the flavor given under low deprivation, whether or not a nutritive solution is used (Experiment 3 ). If flavors are given before and after the daily feeding, rats prefer the flavor they had under high deprivation (before feeding) more if sucrose solutions are used than if saccharin solutions are used and more on a high-deprivation test than on a low-deprivation test (Experiments 1 and 2). It was concluded that the "incentive value" of consumption is not necessarily higher under high deprivation than under low deprivation. The preference for the low-deprivation flavor obtained here may reflect a greater proportional rewarding effect of consumption under low deprivation or may reflect an aversion to the flavor consumed under high deprivation. Perhaps a small taste of flavor under high deprivation initiates responses of digestion that are unsatisfied and thus aversive, and the more so the higher the deprivation level.
\end{abstract}

In a well-known study, Revusky (1967) showed that rats prefer a flavor they previously have consumed under high food deprivation to a flavor consumed previously under low deprivation, whether they were tested under high or low deprivation. This study is often cited in support of the hypothesis that deprivation level affects the incentive value of food (e.g., Bolles, 1970; Revusky \& Garcia, 1970). Because of the theoretical importance of Revusky's finding, it is important to determine the generality of the effect. The purpose of the present experiments was to determine whether rats prefer a flavor previously consumed under high deprivation to one consumed under low deprivation under a wide variety of circumstances.

Revusky (1967) and Revusky and Garcia (1970) suggested that the mechanism underlying rats' preference for the high-deprivation flavor might be an association between the flavor and the long-delayed nutritional aftereffects of food under particular deprivation levels. Revusky (1967) used grape juice and milk, both nutritive liquids. In support of the importance of caloric value, Revusky (1967) cited Smith and Capretta (1956), who found that more errors were made in a T-maze in which correct responses were rewarded with saccharin if the rats had previously consumed saccharin under high deprivation than if they had consumed it under low deprivation.

This research was supported in part by a Purdue Research Foundation David Ross grant. Reprints can be obtained from Elizabeth D. Capaldi, Department of Psychological Sciences, Purdue University, West Lafayette, Indiana 47907.
Revusky (1967) inferred from these data that the preference for nonnutritive foods decreases as a function of the deprivation level at which they were ingested in the past. Because these data are opposite to those obtained by Revusky using nutritive foods, it appears that nutritive value may be an important variable. Experiment 1 sought to determine whether rats' preference for a flavor consumed under high deprivation to one consumed under low deprivation occurs only if the flavors are given in nutritive substances.

\section{EXPERIMENT 1}

In Experiment 1, rats consumed either cinnamonor wintergreen-flavored water, one flavor under high deprivation and the other under low deprivation. For half the rats, the flavor solutions also contained sucrose; for the other half, the flavor solutions contained saccharin. If the preference for a flavor consumed under high deprivation is determined by nutritional aftereffect, this preference might occur only for rats given sucrose solutions and not for those given saccharin solutions.

\footnotetext{
Method

Subjects. The subjects were 20 male albino rats, 188 days old at the beginning of the experiment. They were 92 days old upon arrival at the laboratory from the Holtzman Co., Madison, Wisconsin, and had been previously run in a straight-alley instrumental learning experiment. The rats were distributed evenly into new groups, balancing for group in the previous experiment. Rats had been fed $14 \mathrm{~g}$ of Wayne Lab Blox per day for 93 days prior to Day 1 of Experiment 1.
} 
Materials. Flavored solutions were presented in 50-mi Nalgene centrifuge tubes with rubber stoppers and metal spouts. Flavor cues consisted of $1 \%$ cinnamon ( $2 \%$ imitation cinnamon flavoring mixed with $100 \%$ ethanol) or $1 \%$ wintergreen ( $2 \%$ imitation wintergreen flavoring mixed with $100 \%$ ethanol) dissolved in a saccharin solution with water (.15\% saccharin) or a sucrose-water solution ( $8 \%$ sucrose).

Procedure. All training and testing occurred in the rats' home cages. Ad-lib water was available throughout the experiment in $350-\mathrm{ml}$ brown bottles mounted in the center of the cage front. For 6 days before the experiment began, rats were given Wayne Lab Blox for $1 \mathrm{~h} /$ day beginning at 8:15 a.m. Food was presented and removed at the rate of one cage every $30 \mathrm{sec}$ throughout the experiment. There were 10 days of training and 6 days of testing. On training and testing days, rats were given their food for $75 \mathrm{~min}$ beginning at 9:37 a.m.

Rats were given $5 \mathrm{ml}$ of one flavor solution for $20 \mathrm{~min}$ beginning at 9:07 a.m. before feeding on each of the 10 days of training and $5 \mathrm{ml}$ of the other flavor solution at 11:07 a.m. (15 min after the food was removed). This tube remained on the cage overnight. For half the rats, flavors were given in sucrose solution; for the other half, they were given in saccharin solution. For half the rats that were given sucrose and half those given saccharin, cinnamon (C) was the flavor given before eating and wintergreen (W) was given after feeding; for the other half of the rats, the reverse was true.

Position of the Nalgene tubes relative to the water bottle was reversed every day. Two or three rats in each subgroup began with the tube on the left of the water bottle on the first day of training; the others began with the tube on the right of the water bottle. The Nalgene tube was placed adjacent to the water bottle, so that the spout was about $2 \mathrm{~cm}$ from the floor of the cage and $5 \mathrm{~cm}$ from the side. Of the $5 \mathrm{ml}$ given, the mean amount consumed under high deprivation was $4.42 \mathrm{ml}$ and under low deprivation was $4.97 \mathrm{ml}$; amount consumed did not vary by group or as a function of saccharin vs. sucrose.

Before feeding on each of the 4 test days, rats received two consecutive 10 -min two-bottle choice tests between $40 \mathrm{ml}$ of each of the training solutions. Two more 10 -min tests were given after feeding. The positions of the flavor cues on the first prefeeding and the first postfeeding test were $C$ on the left and $W$ on the right for half the rats and the reverse for the other half of the rats on Days 1 and 4 in each subgroup. These initial positions were the opposite for all rats on Days 2 and 3. All rats began their second prefeeding test and second postfeeding test with flavor cues in the positions opposite to those on the initial prefeeding and initial postfeeding test. Positions were also reversed after $5 \mathrm{~min}$ of each 10-min test. If a rat was drinking at reversal time within the 10 -min test, that tube was removed first and the other tube was put in its place. When drinking resumed, the first tube was placed in the opposite position. If the rat was not drinking, the tube with the lesser amount of solution was removed first, the other tube was then removed, and the tube with the lesser amount of solution was placed in the opposite position, as was the other tube; replacement occurred regardless of the rats' drinking behavior. Presentation of flavors occurred at 1 -min intervals from cage to cage. The prefeeding tests began at 8:45 a.m., and the second 10-min test began 1 min following the presentation of tubes to the 20th rat and was the same as in the first 10-min test. Postfeeding tests began $15 \mathrm{~min}$ after food was removed (at 11:07 a.m.) and were the same as the prefeeding tests.

\section{Results}

Following Revusky (1967), one flavor was chosen arbitrarily, and preference for this flavor was analyzed as the dependent variable. Analyzing amount consumed rather than these preference data produced identical results in all experiments. Preference for wintergreen ( $\mathrm{ml}$ consumed of wintergreen/total $\mathrm{ml}$
Table 1

Mean Preference for Wintergreen for Each Group When Tested Under High and Under Low Deprivation

\begin{tabular}{ccc} 
& \multicolumn{2}{c}{$\begin{array}{c}\text { High Deprivation Cue } \\
\text { in Training }\end{array}$} \\
\cline { 2 - 3 } Test & $\mathrm{C}$ & $\mathrm{W}$ \\
\hline & \multicolumn{2}{c}{ Sucrose Group } \\
High Deprivation & .370 & .471 \\
Low Deprivation & .451 & .366 \\
& \multicolumn{2}{c}{ Saccharin Group } \\
& .442 & .480 \\
High Deprivation & .421 & .406 \\
Low Deprivation & &
\end{tabular}

consumed of wintergreen and cinnamon) for each group is shown in Table 1 on the high-deprivation test and the low-deprivation test summed over the 4 test days. Each row contains data for 10 subjects, five with $\mathrm{C}$ as the high-deprivation flavor and five with $\mathrm{W}$ as the high-deprivation flavor; test deprivation is a within-subjects variable. As is shown in Table 1, the preference for wintergreen was higher if it had been consumed under high rather than low deprivation only when test deprivation was high. When test deprivation was low, the preference for wintergreen was higher if it had been consumed under low deprivation. This was true whether the flavors were dissolved in saccharin or sucrose, although the effect was larger with the sucrose solution.

Analysis of variance substantiated this description. The analysis included between-subjects factors of saccharin vs. sucrose, C vs. W as high-deprivation flavor, and within-subjects factors of test deprivation and days. The only significant effect in the overall analysis was the interaction of high-deprivation flavor with test deprivation $[\mathrm{F}(1,16)=9.39, \mathrm{p}<.01]$. Thus, the preference for $\mathrm{W}$ was higher if it was the highdeprivation flavor when test deprivation was high; when test deprivation was low, the preference for $W$ was higher if it was the low-deprivation cue. A separate analysis of variance on the high-deprivation test alone (using the error term for the high-deprivation data only) indicated that the greater preference for $W$ when it was the high-deprivation flavor was significant $[F(1,16)=4.53, p<.05]$. A similar analysis on the low-deprivation test indicated no significant difference $[F(1,16)=1.63, p>.20]$. Neither difference was significant using the error term from the overall analysis.

In the overall analysis, the high-deprivation flavor $x$ test deprivation interaction did not vary with sucrose vs. saccharin $[F(1,16)=2.92, p>.10]$. Despite this, a separate analysis of variance on the sucrose group alone showed a significant high-deprivation flavor $x$ test deprivation interaction $[F(1,8)=27.93, p<.001]$, while the same analysis on the saccharin group alone produced a nonsignificant interaction. In neither analysis was the main effect of high-deprivation flavor significant $(F s<1)$. Using the overall error term pro- 
duced the same pattern of significant and nonsignificant results [high-deprivation flavor $\times$ test deprivation interaction for the sucrose group: $F(1,8)=11.52$, $\mathrm{p}<.01]$.

\section{Discussion}

Revusky (1967) found that rats preferred a flavor they had consumed previously under high deprivation whether they were tested under high or low deprivation. In Experiment 1, we failed to obtain this result. Rather, a preference for the high-deprivation flavor was found only when testing was given under high deprivation; the preference was in the opposite direction when test deprivation was low. Our results are easily interpreted in terms of associations with deprivation stimuli. If rats associate the consumption of flavors with the deprivation under which the flavors were given in training, then in testing they will show higher consumption of the flavor they were trained with under each deprivation than of the flavor they did not receive under that deprivation. These data are consistent with other data we have presented recently showing strong control over behavior by internal deprivation-related cues (Capaldi \& Davidson, 1979; Capaldi, Viveiros, \& Davidson, 1981). However, in Experiment 1, the association is not directly between the deprivation cue and a response but, rather, between a taste and consumption, conditional on a particular deprivation cue. This type of association has been reported previously (Peck \& Ader, 1974), although there is some difficulty obtaining the effect (Revusky, Pohl, \& Coombes, 1980).

The question remains why the rats in Experiment 1 did not prefer wintergreen more if it was the highdeprivation flavor when they were tested under low deprivation, whereas Revusky (1967) found a greater preference for the high-deprivation flavor when testing occurred under low deprivation. (Actually, in Revusky's 1967 experiment, the preference for the high-deprivation flavor was greater when testing occurred under low deprivation than when testing occurred under high deprivation; this preference was significant when testing was at 0 - or 9 -h deprivation but was not significant at the 23-h test deprivation.) There were many procedural differences between Experiment 1 and Revusky's (1967) experiment that could be responsible for the difference in results. One of these differences is the timing involved. We fed the daily ration $10 \mathrm{~min}$ after removal of the high-deprivation flavor and removed food $15 \mathrm{~min}$ before the low-deprivation flavor was given. Revusky (1967) gave the high-deprivation flavor $135 \mathrm{~min}$ before feeding and the low-deprivation flavor "shortly after" the daily feeding. In both experiments, the role of associations between the flavors and the daily feeding is unclear. For example, in our experiment, it is possible that the rats associated the high-deprivation flavor with some aspect of the subsequent feeding and that this effect reduced any differences due to saccharin vs. sucrose and produced a strong association with the high-deprivation cue. Recent data have shown rats are capable of making associations between a flavor and $20 \mathrm{ml}$ of a $20 \%$ dextrose solution given 30 min later (Holman, 1975). This association was not made if $20 \mathrm{ml}$ of saccharin solution were given, suggesting that the caloric value of sucrose is important in the formation of this association. Perhaps the flavor becomes associated with the nutritional aftereffects of the subsequent substance or perhaps some taste difference as a function of calories is involved in this difference (e.g., Bolles, Hayward, \& Crandall, 1981). The daily feeding in Experiment 1 was, of course, nutritive, so perhaps associations between flavors and some aspect of this feeding produced the results of Experiment 1 rather than the association of flavors with the nutritive aftereffects of the flavored substances themselves. In addition to the highdeprivation flavor's being associated with some aspect of the daily feeding, it is also possible that the lowdeprivation flavor in Experiment 1 and in Revusky's (1967) experiment could be associated with the removal of food rather than with the nutritive aftereffect of consuming a substance under low deprivation. Experiment 2 was a preliminary investigation of the role of associations of taste with some aspect of the daily feeding that came between the two flavors in producing taste preferences.

\section{EXPERIMENT 2}

In Experiment 2, one group received flavors before and after eating, as in Experiment 1 and in Revusky's (1967) experiment. Two other groups received one of their flavors at a time that was neither just before nor just after eating. To accomplish this and to still have a wide range of hunger levels, rats were fed every other day. We have used this procedure successfully previously (Capaldi et al., 1981). Group HL received their first flavor before being fed (high deprivation, $\mathrm{H}$ ) and their second flavor after being fed (low deprivation, L). Group HM received their first flavor before being fed $(\mathrm{H})$, but received their second flavor on days they were not fed. Thus, their second flavor was given under medium deprivation $(\mathrm{M})$ and was not associated with the receipt or removal of food. Group ML received their first flavor on days they were not fed $(\mathrm{M})$ and their second flavor after being fed (L). If preference for the high-deprivation flavor is produced by association of the high-deprivation flavor with some aspect of the subsequent feeding, Groups HL and HM should prefer their higher deprivation flavor, while Group ML should not. If rats avoid flavors associated with the removal of food, Groups HL and ML should avoid the low-deprivation flavor (and thus prefer the high-deprivation flavor for Group HL and the medium-deprivation flavor 
for Group ML), while Group HM should not. If all groups prefer their higher deprivation flavor, this will be ambiguous. This result could mean associations between tastes and some aspect of both the receipt and removal of food are important or that tastes are associated with the effects of the solution under high and low deprivation. Once again, half the rats had their flavors dissolved in sucrose, and half had their flavors dissolved in saccharin.

\section{Method}

Subjects. The subjects were 54 male albino rats, 130 days old at the beginning of the experiment. They were 80 days old upon arrival at the laboratory from the Holtzman Co., Madison, Wisconsin. Thirty of the rats had previously been used in a straightalley instrumental learning experiment, and the other 24 had been in a shuttlebox one-way avoidance learning experiment. The rats were evenly distributed into six new groups of nine subjects each with respect to their former groups and experimenters. All rats had been fed $14 \mathrm{~g}$ of food (Wayne Lab Blox) for at least 30 days before Experiment 2 began.

Materials. The flavor solutions were the same as in Experiment 1.

Procedure. All procedures were the same as in Experiment 1 except as mentioned. Beginning on Day 1 , rats were fed $35 \mathrm{~g}$ on even-numbered days at 1:30 p.m. and were not fed on oddnumbered days. Ad-lib water was available throughout the experiment. Training began on Day 21. As in Experiment 1, half the rats were given flavors dissolved in saccharin, and half had flavors dissolved in sucrose solution (groups referred to as Sac and Suc, respectively). Flavor-solution combinations were balanced across groups; four or five subjects in each group had $C$ as the higher deprivation flavor, and the remainder of each group had $W$ as the high-deprivation flavor.

The six groups were given $5 \mathrm{ml}$ of the appropriate flavor solution under high and low deprivation (Groups HL-Sac and HL-Suc), high and medium deprivation (Groups HM-Sac and HM-Suc), or medium and low deprivation (Groups ML-Sac and ML-Suc). On even-numbered days, the high-deprivation flavor solution was given at 1:00 p.m. (30 min before food) and was removed $20 \mathrm{~min}$ later; the low-deprivation flavor solution was given at 3:20 p.m. (110 min after food was given, food was still present in the cages) and remained on the cages until the next day. For medium deprivation, rats were given their flavor solution at 2:30 p.m. on nonfeeding days (odd-numbered days, $25 \mathrm{~h}$ after they had been fed $35 \mathrm{~g}$ ), and the tube remained on the cage until the next day.

Position of the Nalgene tube containing the flavored saccharin or sucrose solution relative to the water bottle double-alternated over days, beginning with left for half the rats and right for the other half in Groups HM and ML. The same was true in Group HL, except the two consecutive positions occurred on 1 day because this group was trained only on odd-numbered days. Each group had 10 experiences with each flavor-deprivation combination. The mean amount consumed of the $5 \mathrm{ml}$ given under the higher deprivation were: Group $\mathrm{HL}=4.41 \mathrm{ml}$, Group $\mathrm{HM}=4.56 \mathrm{ml}$, and Group $\mathrm{ML}=4.79 \mathrm{ml}$; under the lower deprivation, Group $\mathrm{HL}$ $=4.82 \mathrm{ml}$, Group $\mathrm{HM}=4.82 \mathrm{ml}$, and Group $\mathrm{ML}=4.86 \mathrm{ml}$. As can be seen, less was consumed under high deprivation when only 20-min access was given than under the other deprivations with longer access. The amount consumed did not vary as a function of saccharin vs. sucrose $[F(1,42)=3.52, p>.05]$, and there was no significant interaction with saccharin vs. sucrose.

There were 7 days of testing. On the first 4 days, only a single 10 -min test was given under each deprivation. Each group was tested under each of its training deprivations a total of twice on these 4 days. Testing under $M$ occurred at 2:30 p.m. on Days 1 and 3, testing under $H$ and $L$ occurred on Days 2 and 4 (55 min before the 1:40 p.m. feeding and $1 \mathrm{~h}$ and $50 \mathrm{~min}$ after feeding, respectively). There was a 3-day break between Days 4 and 5 of testing during which the deprivation schedule was maintained. On Days 5, 6, and 7 of testing, two consecutive 10-min tests were given under each deprivation, before feeding $(H)$ on Day $S$, at 2:00 and 2:40 p.m. on Day 6 (a nonfeeding day, M), and after feeding (L) on Day 7. Initial positions of the tubes were counterbalanced as in Experiment 1, and the positions of the tubes were reversed after $S$ min of each test, exactly as in Experiment 1.

\section{Results}

Once again, preference for wintergreen ( $\mathrm{ml}$ consumed of wintergreen/total ml consumed) was computed for the dependent variable. On the first 4 days of testing, only a 10-min test was used. There were no significant differences in these tests. Inasmuch as we had used a 20-min test in Experiment 1 and Revusky (1967) used a 30-min test, on Days 5, 6, and 7 we increased the test length to $20 \mathrm{~min}$ (two consecutive 10-min tests). Reanalysis of Experiment 1 and Experiment 3's data indicated there were also no significant differences if the first 10-min test was analyzed alone. Thus, it takes longer than $10 \mathrm{~min}$ for significant preferences to manifest themselves. Table 2 shows the preferences for each group on the 3 days using $20 \mathrm{~min}$ of testing.

Group HL in Experiment 2 received flavors before and after feeding, just as in Experiment 1 and in Revusky's (1967) experiment, the only difference being the deprivation schedule involved feeding every other day in Experiment 2 rather than every day in Experiment 1 and in Revusky's (1967) experiment. As can be seen in Table 2, in Experiment 2, Group

Table 2

Mean Preference for Wintergreen for Each Group When Tested Under the Higher and Under the Lower Deprivation

\begin{tabular}{ccc} 
& \multicolumn{2}{c}{$\begin{array}{c}\text { High Deprivation Cue } \\
\text { in Training }\end{array}$} \\
\cline { 2 - 3 } Test & $\mathrm{C}$ & $\mathrm{W}$ \\
\hline Higher Deprivation & \multicolumn{2}{c}{ Group HL-Suc } \\
Lower Deprivation & .358 & .601 \\
& .391 & .498 \\
Higher Deprivation & .658 & .618 \\
Lower Deprivation & .530 & .372 \\
& \multicolumn{2}{c}{ Group HM-Suc } \\
Higher Deprivation & .367 & .470 \\
Lower Deprivation & .507 & .447 \\
& \multicolumn{2}{c}{ Group HM-Sac } \\
Higher Deprivation & .594 & .536 \\
Lower Deprivation & .488 & .582 \\
& \multicolumn{2}{c}{ Group ML-Suc } \\
Higher Deprivation & .442 & .452 \\
Lower Deprivation & .336 & .446 \\
& \multicolumn{2}{c}{ Group ML-Sac } \\
Higher Deprivation & .692 & .367 \\
Lower Deprivation & .548 & .482 \\
\hline
\end{tabular}


HL-Suc preferred wintergreen more when it was the high-deprivation flavor than when it was the lowdeprivation flavor, whether testing occurred under high or low deprivation, but the difference was larger on the high-deprivation test than on the low-deprivation test. Thus, we replicated Revusky's (1967) results when conditions were similar to his. When flavors were dissolved in saccharin, Group HL-Sac preferred wintergreen more when it was the low-deprivation flavor when testing occurred under low deprivation and had a slight preference in the same direction when testing occurred under high deprivation. These results are consistent with those of Experiment 1 in suggesting that the preference for the high-deprivation flavor is greater if flavors are given in sucrose rather than in saccharin, and if the test is given under high deprivation rather than under low deprivation. The effect of test deprivation was larger in Experiment 1 than in Experiment 2, and the effect of saccharin vs. sucrose was larger in Experiment 2 than in Experiment 1, but the results in both experiments were in the same direction. It will be recalled that in Experiment 1 the preference for the high-deprivation flavor was greater when flavors were given in sucrose solution than when they were given in saccharin solution, although not significantly greater.

To determine the role of associations of flavors with some aspect of feeding in producing these results, Group HL's data must be compared with those of the other groups. As indicated, when test deprivation was high, Group HL-Suc preferred wintergreen more if it was the high-deprivation flavor. Group HM-Suc did also, but Group ML-Suc had no preference when testing was under the higher deprivation. This finding suggests that associations of the highdeprivation flavor with some aspect of the feeding may play a role in producing a greater preference for the high-deprivation flavor in a test under high deprivation. When test deprivation was low, Group HL-Suc also preferred wintergreen more if it was the highdeprivation flavor. Group ML-Suc did also, but Group HM-Suc did not. This suggests that receipt of the low-deprivation flavor shortly after feeding may be playing a role in producing a greater preference for the high-deprivation flavor in a test given under low deprivation.

Considering the saccharin groups, on the higher deprivation test, all three groups preferred wintergreen more if it was the low-deprivation flavor, although this effect was large only for Group ML. This effect may thus not be strongly affected by associations with feeding. In the low-deprivation test for the saccharin groups, a greater preference for wintergreen occurred if it was the low-deprivation flavor for Groups HL-Sac and ML-Sac but not for Group HM-Sac. It appears that receiving a flavor shortly after feeding may play a role in producing this effect.
Analysis of variance included the same factors as in Experiment 1 plus the between-groups deprivation differences (HL vs. HM vs. ML). This analysis showed a significant high-deprivation flavor $(\mathrm{C}$ or $\mathrm{W}) \times$ caloric value interaction $[F(1,42)=7.33, p<.01]$. Summing over all groups with sucrose, the preference for $\mathrm{W}$ was greater if it was the high-deprivation flavor; with saccharin, preference for $\mathrm{W}$ was greater if it was the low-deprivation flavor. This effect did not interact significantly with the between-groups deprivation differences ( $\mathrm{HL}$ vs. $\mathrm{HM}$ vs. $\mathrm{ML}$ ) $[\mathrm{F}(2,42)=1.14$, $\mathrm{p}>.30]$ or with deprivation on the test $[\mathrm{F}(1,42)=1.23$, $\mathrm{p}>.20$, despite the trends described above.

Subsequent analyses were done on subsets of the data. Analyzing the sucrose data alone (using the error term for the sucrose data only) showed a significant preference for the high-deprivation flavor over all three groups $[\mathrm{F}(1,21)=4.62, \mathrm{p}<.05]$, while a similar analysis on the saccharin data alone showed no significant preference. Analyzing each group's data alone showed a significant preference for the high-deprivation flavor for Group HL-Suc, but no significant preferences for Groups HM-Suc or ML-Suc, and a significant preference for the low-deprivation flavor for Group ML-Sac, but no significant preferences for Group HL-Sac or HM-Sac. If the overall error term was used in these analyses, the only significant difference of those mentioned in this paragraph was Group ML-Sac's preference for the low-deprivation flavor.

\section{Discussion}

In Experiment 2, Group HL-Suc, given flavors before and after a feeding dissolved in sucrose, preferred wintergreen more if it was the high-deprivation flavor, whether testing occurred under high or low deprivation (although the difference was larger on the high-deprivation test, as in Experiment 1). This group is closest to Revusky's (1967) conditions in that flavors in caloric solutions were given before and after feeding. Consistent with Revusky's hypothesis that caloric value might be important, this effect did not occur when saccharin was used. It is also interesting to note that when saccharin was used, a preference (not significant) for the low-deprivation flavor occurred for Group HL-Sac. It seems that receiving saccharin under high deprivation may be aversive. This would be consistent with the idea that preferences are produced by calories' varying in incentive value as a function of hunger and would extend this notion to include the idea that consumption of noncaloric substances when hungry can be aversive.

However, a tendency toward a greater preference for the high-deprivation flavor dissolved in sucrose did not occur in a high-deprivation test when the higher deprivation flavor and test were given separately from feeding (Group ML-Suc), and did not occur in a low- 
deprivation test when the low-deprivation flavor and test were given separately from feeding (Group HM-Suc). These data suggest that associations of flavors with some aspect of feeding may be important in producing these preference data, rather than association of the consequences of consuming the flavors themselves as a function of hunger level during consumption producing the effects. Experiment 3 was run to evaluate this possibility. The purpose of Experiment 3 was to measure flavor preferences as a function of hunger level during flavor consumption when flavor consumption was totally separated from feeding of the food ration.

\section{EXPERIMENT 3}

In addition to giving flavors under different deprivation levels separately from the daily feeding, the type of cue associated with consumption under each deprivation level was also varied in Experiment 3. Some groups had flavors under different deprivation levels, while others had their solutions in a bottle in a particular position under each deprivation level.

Position was investigated in addition to flavors because Holman (1980) reported that what was learned in cue-incentive pairings when different positions were associated with different incentives differed from what was learned when different flavors were associated with different incentives. However, Kurtz and Jarka (1968), using position cues rather than flavor cues, reported findings similar to Revusky's (1967), suggesting position cues and flavor cues produce similar effects when preference between cues as a function of hunger level during original training is measured. Kurtz and Jarka reported that rats preferred the arm of a T-maze in which they found food under higher hunger whether tested under high or low hunger. Kurtz and Jarka (1968) interpreted their results to indicate that the incentive value of food is greater under high hunger than under low hunger. Kurtz and Jarka ran subjects that were 23 -h food deprived or nondeprived, and removed or gave food $30 \mathrm{~min}$ following a trial. Also, a nutritive reward was used. Thus, it is not known whether Kurtz and Jarka's results would occur with nonnutritive rewards or whether associations with some aspect of the feeding were playing a role in their finding. In Experiment 3, the effects of position and flavor cues were directly compared to determine whether both cues produce similar preferences as a function of hunger level during original consumption. Also, solutions were given separately from feeding, making the association of flavors or positions with any aspect of the feeding unlikely. Finally, half the rats had sucrose solutions and half had saccharin solutions.

\section{Method}

Subjects. The subjects were 40 male albino rats, 149 days old at the beginning of Experiment 3. They were 80 days old upon arrival at the laboratory from the Holtzman Co., Madison, Wisconsin, and had previously been in a straight-alley instrumental learning experiment and on a 14-g day food-deprivation schedule for at least 30 days. The rats were evenly distributed into new groups with respect to their former groups and experimenters. All rats had received ad-lib food and water for 10 to 13 days prior to the beginning of Experiment 3.

Materials. The flavor solutions were the same as in Experiments 1 and 2. For the position cue, the Nalgene tube was placed on the lower left (approximately $1 \mathrm{~cm}$ from the left side and $1 \mathrm{~cm}$ from the bottom of the cage) or upper right (approximately $1 \mathrm{~cm}$ from the right side and $10-11 \mathrm{~cm}$ from the bottom of the cage) of the cage front.

Procedure. All procedures were the same as in the preceding experiments except as mentioned. Rats were fed $35 \mathrm{~g}$ on evennumbered days at 6:30 p.m. All training and testing occurred at 9:30 a.m. Low-deprivation training and testing were given on oddnumbered days (about $14 \mathrm{~h}$ after food was given), and highdeprivation training and testing were given on even-numbered days (about $38 \mathrm{~h}$ after food was given). On odd-numbered days, body weights were approximately $90 \%$ of ad-lib weights (varying between $87 \%$ and $93 \%$ ), and on even-numbered days body weights were about $82 \%$ of ad-lib weights (varying from $80 \%$ to $84 \%$ ). These percentages did not vary by group. Half the rats received flavored solutions just as in the previous experiments, $\mathrm{C}$ or $\mathrm{W}$ in saccharin or sucrose solution. The other half had the Nalgene tube containing unflavored solutions always in a particular position under a particular deprivation; the position-deprivation combinations were balanced in the same way as the flavor-deprivation combinations in all experiments. Half of each of these groups were given saccharin solution, and the other half had sucrose. For rats given position cues, the solutions were unflavored (i.e., no $\mathrm{C}$ or $\mathrm{W}$ was added). For all rats, $5 \mathrm{ml}$ of the appropriate solutions were given, and the bottle remained on the cage until the next day. All rats consumed the entire solution.

Four days of testing followed training. Days 1 and 3 of testing were low-deprivation days, and Days 2 and 4 were high-deprivation days. On Days 1 and 2 of testing, rats were presented with a twobottle choice between $40 \mathrm{ml}$ of their training solutions for $20 \mathrm{~min}$. For flavor groups, the positions of the two tubes were reversed every $5 \mathrm{~min}$, as in the preceding experiments. Because some rats consumed all $40 \mathrm{ml}$ of one of the test solutions, testing on Days 3 and 4 reverted to our former procedure of two $10-\mathrm{min}$ choice tests -Test 2 began 55 min after Test 1 had begun.

\section{Results}

Preferences for $\mathrm{W}$ (for the flavor groups) and for the right side (for the position groups) are shown in Table 3 in the high-deprivation test and the lowdeprivation test.

Table 3

Mean Preferences for Wintergreen (Flavor Groups) or Right Side (Position Groups) When Tested Under High and Under Low Deprivation

\begin{tabular}{cccccc} 
& \multicolumn{4}{c}{ High Deprivation Cue in Training } \\
\cline { 2 - 6 } Test & \multicolumn{2}{c}{ Flavor Groups } & \multicolumn{3}{c}{ Position Groups } \\
\cline { 2 - 6 } & $\mathrm{C}$ & $\mathrm{W}$ & Left & Right \\
\hline & \multicolumn{5}{c}{ Sucrose } \\
High Deprivation & .515 & .359 & .163 & .295 \\
Low Deprivation & .461 & .438 & .165 & .243 \\
& \multicolumn{5}{c}{ Saccharin } \\
High Deprivation & .561 & .256 & .331 & .491 \\
Low Deprivation & .552 & .273 & .428 & .585 \\
\hline
\end{tabular}


As can be seen in Table 3, the preference for $W$ was higher for the flavor groups if $\mathrm{W}$ had been given under low deprivation rather than high deprivation for both saccharin and sucrose groups on the highdeprivation and the low-deprivation test. Under no conditions did the flavor groups show a greater preference for $\mathrm{W}$ when it was the high-deprivation flavor. In contrast, the position group's preference for right was greater if they had been given their solution on the right under high deprivation rather than low deprivation, whether they had been given unflavored sucrose or saccharin and whether the test was given under high or low deprivation.

Analysis of variance showed a significant highdeprivation cue $\times$ flavor-position interaction $[F(1,32)$ $=12.74, \mathrm{p}<.01]$. This interaction did not depend on whether saccharin or sucrose solutions were given $[F(1,32)=2.03, p>.10]$ or on whether a highdeprivation or low-deprivation test was used $(F<1)$.

When data for flavor groups were analyzed alone, preference for $\mathrm{W}$ was significantly greater when it was the low-deprivation cue than when it was the highdeprivation cue $[F(1,16)=8.90, p<.01]$. The interaction of this preference with saccharin vs. sucrose was not significant $[F(1,16)=2.53, p>.10]$, and this preference did not interact with test deprivation $[F(1,16)=1.04]$. For the position groups, preference for the right side was greater if the solution was in that position under high deprivation rather than low deprivation. This effect just missed significance for saccharin and sucrose groups combined. Although the interaction of this preference with saccharin vs. sucrose was not significant $(F<1)$, the preference for the right side was significantly greater if the solution was in that position under high deprivation rather than low deprivation for saccharin groups alone $(p<.05)$ but not for the sucrose groups alone. All these results were the same whether the overall error term or individual error terms were used.

\section{Discussion}

When all associations between flavors and any aspects of feeding were made unlikely by separating flavors from feeding by long time intervals, rats preferred the flavor they consumed under low deprivation, whether tested under high or low deprivation and whether flavors were given with sucrose or saccharin. It seems that the preferences for the high-deprivation flavors obtained in Experiments 1 and 2 must have been produced by the association of flavors with some aspects of receipt or removal of food.

In contrast, rats preferred the tube position they had under high deprivation, at least with saccharin solutions. This latter finding can be interpreted as reflecting the conditioning of an instrumental response of approaching each tube location, with conditioning of this response being stronger the higher the deprivation was under which conditioning had occurred.
There is ample evidence that conditioning of instrumental responses is stronger when carried out under high deprivation than under low deprivation (e.g., Capaldi \& Hovancik, 1973; Eisenberger, Myers, \& Kaplan, 1973). If this interpretation is correct, it implies that preference data measuring responses that were made in training can be confounded by differential strength of conditioning of those responses. For example, in the Kurtz and Jarka (1968) study described above, the rats approached the high-deprivation side of the T-maze only under high deprivation in training and approached the low-deprivation side only under low deprivation in training. Thus, stronger conditioning of the approach response under high deprivation than under low deprivation can account for the Kurtz and Jarka data, and no preference need be postulated. The same suggestion has been made by Eisenberger et al. (1973).

If this interpretation of the position data is made, the question remains why consumatory responses are not more strongly conditioned under high deprivation than under low deprivation. If consumatory responses were more strongly conditioned under high deprivation than under low deprivation, groups trained to drink a flavor under high deprivation would drink more of that flavor later than they would drink of a flavor given under low deprivation. However, the present experiments have shown that they do not. Thus, if the results of Experiment 3 for the position cue are to be interpreted in terms of strength of conditioning of responses as a function of deprivation level during training, it must be assumed that instrumental responses are more strongly conditioned under high deprivation than under low deprivation, whereas consumatory responses are not. An alternative explanation of Experiment 3's results could relate the differences in the effects of flavor cues and position cues to when the cues are received relative to the drinking response being measured. Position cues are responded to and thus received before the rat drinks, whereas the flavor cues are not received until after the rat drinks. Wilson, Sherman, and Holman (1981) recently showed that a taste aversion to sucrose has different effects on performance to a cue associated with sucrose depending on whether the cue is presented before the response being measured (as a discriminative stimulus) or after the response (as a secondary reinforcer). Perhaps the difference here between position cues and taste cues is determined by their temporal relationship to the drinking response being measured. We are currently investigating this hypothesis.

\section{GENERAL DISCUSSION}

The preference for a flavor previously consumed under high deprivation obtained by Revusky (1967) 
does indeed occur when conditions are similar to those Revusky (1967) employed. Group HL in Experiment 2 given sucrose solutions showed a greater preference for wintergreen if it was the high-deprivation flavor than if it was the low-deprivation flavor, whether testing was given under high or low deprivation, just as Revusky reported. However, the other groups in Experiment 2 and the results of Experiment 3 show that this result does not occur when flavors are given separately from feeding. In Experiment 2, the greater preference for the high-deprivation flavor was significant only for the group given sucrose solutions before and after feeding. In Experiment 3, when flavors were given in sucrose separately from feeding, preference for wintergreen was greater if it was the lowdeprivation flavor.

These results thus indicate that to the extent the rats' preference for flavors is determined by the deprivation level under which the flavors were consumed, they prefer the low-deprivation flavor. This preference can be overridden when flavors are given closely before or after feeding if the flavors are given in sucrose.

We cannot determine from the present results whether the rats' preference for the flavor they had under low deprivation in Experiment 3 reflects a conditioned preference for the flavor they had under low deprivation, a conditioned aversion to the flavor they had under high deprivation, or both. It may be that a small taste when hungry is aversive; perhaps it initiates responses of digestion (salivation, insulin release, etc.) that are then unsatisfied, intensifying the unpleasantness of hunger, the more so the higher the base level of hunger is. Alternatively, perhaps a small taste of food is more reinforcing proportionately under low deprivation than high deprivation. Although we do not know what produces the preference obtained here for the low-deprivation flavor, we can say that the incentive value of consumption is not always greater under high deprivation than under low deprivation.

These results do not in any way disconfirm Revusky's (1967) hypothesis that rats can associate tastes of food with delayed nutritional aftereffects of the food or Revusky and Garcia's (1970) theory of hunger. Our hypothesis that a small amount of food may actually be aversive under high hunger can be viewed as an extension of Revusky's ideas. We are suggesting that the delayed aftereffect of ingestion under high hunger is not always pleasant and may not be related directly to hunger level but to the amount of food relative to the degree of hunger. Our results do suggest, though, that Revusky's (1967) results are probably not attributable to association of the tastes of grape juice and milk with the delayed nutritional aftereffects of grape juice and milk. However, within Revusky's (1967) own hypothesis, it is not surprising that the associations between consumption of grape juice or milk and their nutritional aftereffects would be difficult under his conditions. It would be difficult for the rat to discriminate the nutritional or other aftereffects of $5 \mathrm{ml}$ of grape juice given $135 \mathrm{~min}$ before 75-min access to food, the nutritional or other aftereffects of the meal itself, and the nutritional or other aftereffects of $5 \mathrm{ml}$ of milk given after the 75 -min meal. The present results suggest that, under these conditions, the taste of small amounts of food given before or after feeding become associated with some aspect of the feeding, perhaps its nutritional aftereffects.

However, the present results also suggest that rats do indeed associate tastes of food with the consequences of these foods under different degrees of hunger, as suggested by Revusky (1967) and Revusky and Garcia (1970). Rats preferred their low-deprivation flavor when associations with feeding were made unlikely. Future research can be directed to the determinants of this effect. For example, it appears that rats' preference for the flavor consumed under high deprivation given shortly before feeding is greater when sucrose rather than saccharin solutions are used (Experiments 1 and 2). For some reason, associations of a flavor with some aspect of the daily feeding (perhaps the delayed nutritional aftereffects) are stronger if the flavor is given in sucrose rather than saccharin. This may be related to the difference in nutritive value between sucrose and saccharin. Perhaps the association of the taste with some aspect of the daily feeding is mediated by common nutritional aftereffects. Alternatively, the sucrose vs. saccharin differences could be produced by the different tastes of these substances. Also, it seems that preference for the high-deprivation flavor given before feeding is greater if the test is given under high deprivation than under low deprivation (Experiments 1 and 2), indicating that deprivation stimuli are important in the associations being formed, as they should be within Revusky and Garcia's (1970) theory. Exactly how deprivation stimuli are involved in the associations being formed remains to be untangled. These and other questions are opened by realization that the incentive value of food is not always greater under high deprivation than under low deprivation and that associations involving deprivation stimuli, flavor cues, other cues (such as position), and the food the rat eats throughout the day are all involved in determining later food preferences between cues experienced previously under different hunger levels.

\section{REFERENCES}

Bolles, R. C. Interactions with motivation. In M. H. Marx (Ed.), Learning: Interactions. London: Macmillan, 1970.

Bolles, R. C., Hayward, L., \& Crandall, C. Conditioned taste preferences based on caloric density. Journal of Experimental Psychology: Animal Behavior Processes, 1981, 7, 59-69. 
Capaldi, E. D., \& Davidson, T. L. Control of instrumental behavior by deprivation stimuli. Journal of Experimental Psychology: Animal Behavior Processes, 1979, 5, 355-367.

Capaldi, E. D., \& Hovancik, J. R. Effects of previous body weight level on rats' straight-alley performance. Journal of Experimental Psychology, 1973, 97, 93-97.

Capaldi, E. D., Viveiros, D. M., \& Davidson, T. L. Deprivation stimulus intensity and incentive factors in the control of instrumental responding. Journal of Experimental Psychology: Animal Behavior Processes, 1981, 7, 140-149.

Eisenberger, R., Myers, A. K., \& Kaplan, R. M. Persistent deprivation-shift effect opposite in direction from incentive contrast. Journal of Experimental Psychology, 1973, 99, 400-404.

Holman, E. W. Immediate and delayed reinforcers for flavor preferences in rats. Learning and Motivation, 1975, 6, 91-100.

Holman, E. W. Irrelevant-incentive learning with flavors in rats. Journal of Experimental Psychology: Animal Behavior Processes, 1980, 6, 126-136.

Kurtz, K. H., \& JARKA, R. G. Position preference based on differential food privation. Journal of Comparative and Physiological Psychology, 1968, 66, 518-521.

Peck, J. H., \& ADER, R. Illness-induced taste aversion under states of deprivation and satiation. Animal Learning \& Behavior, 1974, 2, 6-8.

Revusky, S. H. Hunger level during food consumption: Effects on subsequent preference. Psychonomic Science, 1967, 7, 109110.

Revusky, S., \& Garcia, J. Learned associations over long delays. In G. H. Bower (Ed.), The psychology of learning and motivation: Advances in research and theory (Vol. 4). New York: Academic Press, 1970.

Revusky, S., Pohl, R. W., \& Coombes, S. Flavor aversions and deprivation state. Animal Learning \& Behavior, 1980, 8, 543-549.

Smith, M. P., \& Capretta, P. J. Effects of drive level and experience on the reward value of saccharin solutions. Journal of Comparative and Physiological Psychology, 1956, 49, 553-557.

Wilson, C. L., Sherman, J. E., \& Holman, E. W. Aversion to the reinforcer differentially affects conditioned reinforcement and instrumental responding. Journal of Experimental Psy. chology: Animal Behavior Processes, 1981, 7, 165-174.

(Manuscript received August 11, 1981;

revision accepted for publication November 3,1981 .) 